\title{
Consensus guidelines, algorithms and care of the individual patient with type 2 diabetes
}

\author{
J. J. Nolan
}

Received: 18 March 2010 / Accepted: 23 March 2010/Published online: 25 April 2010

(C) Springer-Verlag 2010

Keywords Algorithms · Consensus guidelines · Diabetes · Oral pharmacological agents

Despite advances in understanding of the pathophysiology of type 2 diabetes and the abundance of expert reviews on its treatment, a large gap remains between what is generally believed and what is actually known about treatments for diabetes and their long-term effects. Over a relatively short recent interval the ADA and the EASD have jointly published successive consensus algorithms for the management of hyperglycaemia in patients with type 2 diabetes [1, 2]. The authors of both the 2006 and 2009 consensus algorithms acknowledged in their introductions that while numerous reviews on the treatment of type 2 diabetes have been published in recent years, 'practitioners are often left without a clear pathway of therapy to follow' $[1,2]$. The most recent algorithm, with its two-tiered approach to metabolic management, has clearly not solved this problem and has itself provoked further debate and commentary [3, 4], including most recently the critique by Schernthaner and colleagues [5] published in the current issue of Diabetologia.

The most recent contributors, rather than proposing a further, newer algorithm for diabetes treatment (perhaps we should be grateful that further guidelines have not been proposed), take issue with the 'expert opinion' approach adopted by the ADA/EASD authors and argue instead for an evidence-based strategy, taking into account the impact of various treatments on clinically important endpoints (such as macrovascular events). They put forward the case

J. J. Nolan $(\bowtie)$

Metabolic Research Unit, St James Hospital, Trinity College, Dublin 8, Ireland

e-mail: jnolan@stjames.ie for individual optimisation of care of the patient with the goals of sustained blood glucose control and the reduction of complications and cardiovascular risk. Clearly no sensible clinician would dispute this case, and this includes those who have generated the previous guidelines and algorithms. Following the ancient principle of 'first, do no harm', the overall goal for a good doctor must be the improved care and quality of life of the individual patient. However, equally clearly there is a problem with treatment algorithms for type 2 diabetes based on 'expert opinion'. The article by Schernthaner and colleagues [5] raises some important limitations inherent in this approach and, in the process, points to some of the broader challenges in modern diabetes care. Their conclusion is that the 2009 treatment algorithm is not in fact based on evidence. This debate is useful for those who care for patients with diabetes, in that it emphasises the importance of evidence, while drawing our attention to the wide areas where the answers are not yet known, and where the evidence does not yet exist and may not exist for some time. The prevalence of diabetes continues to increase and actual treatment decisions and choices face individual patients and their doctors on a daily basis. How these decisions are best made in the real world may ultimately be through a pragmatic individual patient plan, taking account of the best available evidence, the stage in life of the patient, the biological stage of progression of their diabetes and the relative priorities of the competing targets and goals in diabetes care (see Fig. 1).

Schernthaner and his colleagues have approached diabetes treatment options under three main themes: (1) glucose lowering, (2) cardiovascular effects and outcomes and (3) pathophysiology and other relevant clinical effects. In relation to glucose lowering, the authors directly challenge the approach taken in the ADA/EASD algorithm. They point out that the short-term efficacies of the available 


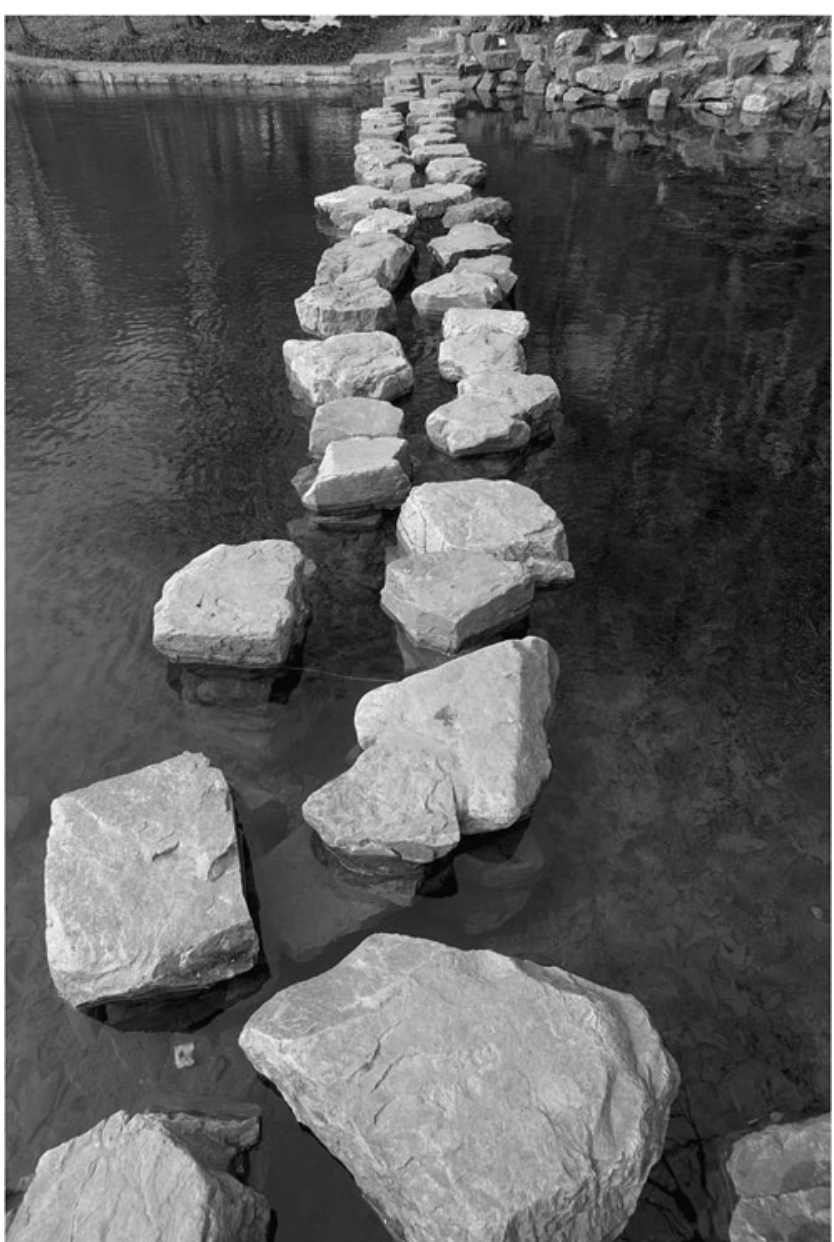

Fig. 1 Individual treatment choices for type 2 diabetes are pragmatic, like the stepping stones across this river

agents (metformin, sulfonylureas, thiazolidinediones, as well as probably glinides and incretin-based agents) are, in fact, similar and therefore not the most important basis on which to choose a treatment. They emphasise the question of longer-term glucose control, a theme which has been addressed in relatively few of the randomised controlled clinical trials. Type 2 diabetes progresses inexorably in all patients, and many of the currently available treatments (with the exception perhaps of metformin) lose their effectiveness over time. Also emphasised in treatment choice are the unwanted effects of hypoglycaemia risk and weight gain. The ADA/EASD algorithm promotes insulin as an early option in their first tier of treatments. Schernthaner and colleagues offer a critique of insulin therapy in the current article, citing recent literature; however, their overall conclusion is that the optimal approach for the use of insulin in type 2 diabetes remains unclear. Long-term cardiovascular risk vs benefit of diabetes treatments is an increasingly important criterion, not only for new drug approval but also for the consideration of clinicians and patients. Under this increasingly controversial theme, the current authors make the case for a more focused consideration of the cardiovascular effects of individual drugs (rather than for glucose lowering per se, for which the existing evidence is much less clear). The difficulty here for those treating patients is that high-quality long-term evidence of this kind is lacking for most of the treatments on offer. The current authors are critical of the ADA/EASD's 'relegation' of rosiglitazone under this heading (as well as the topic of its effects on pathophysiology and its proven durability of glucose-lowering effect). Paradoxically, following meta-analytic data suggesting an increased cardiovascular risk with rosiglitazone [6], this class of agents has now undergone more rigorous long-term cardiovascular study, and the overall conclusion is that the long-term risk-benefit profile of these drugs is uncertain. The problem for newer agents such as incretin-based treatments, which have shown very promising effects in animal studies $[7,8]$ and surrogate effects in clinical trials [9], is that solid evidence from long-term clinical trials will not be available for several years, as is the case with any newer agent that appears on the horizon.

The third theme addressed by Schernthaner and colleagues is the effect of individual treatments on components of the underlying pathophysiology of type 2 diabetes. The relentless progression of the disease is best illustrated by the continuous decline in pancreatic insulin secretion over time. The longest studied agents, metformin and sulfonylureas, cannot halt this process, and whereas metformin itself is neutral in terms of insulin secretion, sulfonylureas are clearly associated with predictable treatment failure over time [10]. On the other hand, the thiazolidinediones and the incretin-based treatments have a more favourable effect on beta cell function, but sufficiently long-term studies of these effects are not yet available. Similarly, the different treatment options have a wide variety of effects on the standard cardiovascular disease risk markers including the lipid profile, markers of inflammation, blood pressure and obesity. The current authors make the case that consideration of the impact of individual drugs on these risk factors is warranted, in the absence of primary endpoint outcomes data. Apart from cardiovascular effects, consideration of other important adverse effects is also a major component of the individual care of patients. The authors re-iterate the importance of unwanted hypoglycaemia, weight gain, fracture risk (with thiazolidinediones), gastrointestinal side effects and the emerging association between diabetes, its treatments and risk of cancer [11].

What lessons can be learnt from this critical analysis, and why is it proving so difficult to map a general pathway for the treatment of type 2 diabetes? Several broader issues are raised that contribute to the 'big picture' and that are directly relevant to the care of patients. The first is the role of consensus guidelines and treatment algorithms them- 
selves. The limits of the 'expert opinion' approach are plain to see, and rightly criticised by Schernthaner and colleagues. However, the alternative extreme, which would insist on only long-term randomised controlled trial-based evidence (because so much is lacking in the field of diabetes), could paralyse clinicians completely and create a new level of despondency and inertia in patient care. However, the diabetes epidemic is now, and treatment decisions for a variety of different patients at different stages of life and disease are required today. Even in relation to long-term glycaemic control, recent evidence makes it clear that overall mortality may be increased in many patients at $\mathrm{HbA}_{1 \mathrm{c}}$ below 7.5\% [12]. However, early diagnosis and early treatment to target newly diagnosed patients before complications may still yield excellent long-term results, as illustrated by the UK Prospective Diabetes Study.

Very few clinicians in practice rely on guidelines and treatment algorithms for treatment decisions. Diabetes care is in fact individualised along much more pragmatic lines, including such issues as patient education, likelihood of compliance, side effects and costs of drugs and the awful burden of polypharmacy. Could diabetes treatment be individualised and personalised along better lines? A wide variety of evidence indicates that this is possible. A more accurate phenotyping of the patient and staging of the state of progression of their disease in terms of beta cell reserve and insulin sensitivity is becoming a reality, and this could much better inform the choice of initial and subsequent treatments. Patients with later-stage disease may benefit from much less stringent glucose control than hitherto believed [12], permitting a better focused and targeted approach to the busy practice or clinic. The best clinical care will of course be founded on evidence but it will also be informed by consideration of the unique circumstances of the individual in whom the exception may sometimes be the rule.

\section{References}

1. Nathan DM, Buse JB, Davidson MB et al (2006) Management of hyperglycaemia in type 2 diabetes: a consensus algorithm for the initiation and adjustment of therapy. A consensus statement from the American Diabetes Association and the European Association for the Study of Diabetes. Diabetologia 49:1711-1721

2. Nathan DM, Buse JB, Davidson MB et al (2009) Management of hyperglycaemia in type 2 diabetes mellitus: a consensus algorithm for the initiation and adjustment of therapy. A consensus statement from the American Diabetes Association and the European Association for the Study of Diabetes. Diabetologia 52:17-30

3. De Fronzo RA (2009) From the triumvirate to the ominous octet: a new paradigm for the treatment of type 2 diabetes mellitus. Diabetes 58:773-795

4. Woo V (2009) Important differences: Canadian Diabetes Association (2008) clinical practice guidelines and the consensus statement of the American Diabetes Association and the European Association for the Study of Diabetes. Diabetologia 52:552-553

5. Schernthaner G, Barnett AH, Betteridge DJ, et al. (2010) Is the ADA/EASD algorithm for the management of type 2 diabetes (January 2009) based on evidence or opinion? A critical analysis. Diabetologia. doi:10.1007/s00125-010-1702-3

6. Nissen SE, Wolski K (2007) Effect of rosiglitazone on the risk of myocardial infarction and death from cardiovascular causes. N Engl J Med 356:2457-2471

7. Timmers L, Henriques JP, de Kleijn DP et al (2009) Exenatide reduces infarct size and improves cardiac function in a porcine model of ischemia and reperfusion injury. J Am Coll Cardiol 53:501-510

8. Noyan-Ashraf MH, Momen MA, Ban K et al (2009) The GLP1 R agonist liraglutide activates cytoprotective pathways and improves outcomes following experimental myocardial infarction in mice. Diabetes 58:975-983

9. Inzucchi SE, McGuire DK (2008) New drugs for the treatment of diabetes: part II-incretin-based therapy and beyond. Circulation 117:574-584

10. Kahn SE, Haffner DM, Heise MA et al (2006) Glycemic durability of rosiglitazone, metformin, or glyburide monotherapy. N Engl J Med 355:2427-2443

11. Currie CJ, Poole CD, Gale EA (2009) The influence of glucoselowering therapies on cancer risk in type 2 diabetes. Diabetologia 52:1766-1777

12. Currie CJ, Peters JR, Tynan A et al (2010) Survival as a function of $\mathrm{HbA1c}$ in people with type 2 diabetes: a retrospective cohort study. Lancet 375:481-489 